\title{
Fibrinogen-Detectable Thrombosis in the Legs and Pulmonary Embolism
}

\author{
N. L. BROWSE, G. CLEMENSON, D. N. CROFT
}

British Medical fournal, 1974, 1, 603-604

\section{Summary}

Thrombosis in the deep veins of the legs commonly follows surgical operations but is often undiagnosed. The fibrinogen uptake test is an aid to its detection. Perfusion lung scanning after injection of labelled macroalbumin aggregates has advanced the diagnosis of pulmonary embolism.

Studies were carried out on $\mathbf{4 0}$ patients undergoing surgical operation to determine whether the results of the fibrinogen uptake test were correlated with those of ventilation-perfusion lung scanning. A statistically significant relationship was found: $18 \%$ of patients had small, clinically silent emboli.

\section{Introduction}

A feature of all necropsy studies on patients who die after surgery is the frequent discovery of unsuspected deep vein thrombosis and pulmonary embolism. For example, Sevitt and Gallagher (1961) showed that $90 \%$ of the patients who died between 8 and 14 days after fracturing the neck of the femur had thrombosis in the deep veins of their lower limbs, and Wessler (1962) found pulmonary emboli in $70 \%$ of an unselected series of patients at necropsy.

The fibrinogen uptake test (F.U.T.) for the diagnosis of deep vein thrombosis brought to the bedside an awareness of the frequency of this disease (Flanc et al., 1968; Negus et al., 1968). Studies in English hospitals have shown that deep vein thrombosis occurs in $25-30 \%$ of all patients undergoing surgery. So far there has been no correlation between the small thrombi detected in the leg by the F.U.T., which here we call F.U.T. thrombi, and pulmonary embolism. This is due to the difficulty of diagnosing pulmonary embolism during life and the natural reluctance to use frequent arteriography solely for research.

Fortunately the diagnosis of pulmonary embolism has been greatly advanced by the development perfusion lung scanning after the injection of labelled macroalbumin aggregates. As this technique requires no more than a single intravenous injection and scanning with a scintiscanner or gamma camera and gives less radiation than one chest $x$-ray exposure it is a suitable research tool. A perfusion defect associated with a normal chest $x$-ray picture is good evidence of pulmonary embolism, but false positive results do occur. In particular, defects of perfusion may arise secondary to defects of ventilation, as in asthma and bronchitis. This problem has been overcome by combining a xenon ventilation scan with a perfusion scan (Williams et al., 1974). Using this technique after surgery we have found the incidence of perfusion defects $(18 \%)$ to correspond to that found in the necropsy studies mentioned above, and we believe this technique to be the most accurate method available.

St. Thomas's Hospital, London SE1 7EH

N. L. BROWSE, M.D., F.R.C.S., Professor of Vascular Surgery

G. CLEMENSON, Research Technician

D. N. CROFT, D.M., M.R.C.P., Consultant Physician
We are studying a method of prophylaxis against deep vein thrombosis and pulmonary embolism using preoperative and postoperative lung scanning to diagnose pulmonary embolism. A preliminary review of the results disclosed a definite correlation between the presence of F.U.T. thrombi in the calf and pulmonary embolism diagnosed by combined ventilationperfusion lung scanning. As this correlation has important clinical implications our figures are presented in this preliminary report. The result of the prophylaxis trial is not presented because the study is not yet complete.

\section{Patients}

Altogether 40 patients were studied ( 21 men and 19 women); 27 had upper abdominal operations, 8 had lower abdominal operations, and 5 had surgery to the head, neck, or chest wall. All were over age 40 or postmenopausal and without severe hypertension or liver disease. The nature and effects of the investigations were explained in detail to each patient and their written consent was obtained. A record was kept of any pyrexia, pain in the chest, sputum, haemoptysis, breathlessness, and any physical signs which indicated the presence of pulmonary or postoperative lung infection.

\section{Methods}

Fibrinogen Uptake Test.-The technique used was that described by Negus et al. (1968); its validity has been proved (Negus et al., 1968; Browse et al., 1971).

Combined Lung Perfusion and Ventilation Imaging.-Combined ventilation and perfusion lung images were obtained using a Nuclear Enterprises gamma camera with a high sensitivity diverging collimator designed for the purpose Ahmad and Clapham, 1974). Ventilation images were obtained after inhalation of a bolus of $3-5 \mathrm{mCi}$ of ${ }^{133} \mathrm{Xe}$, and perfusion images after intravenous injection of $1 \mathrm{mCi}$ of $99 \mathrm{mTc}$ macroaggregated albumin. Anterior posterior and both lateral views were obtained for perfusion studies. For ventilation studies posterior views were taken. Each study was completed within 20-30 minutes. Full details of the technique and a clinical assessment of the results are given in the previous paper (Williams et al., 1974). Lung perfusion and ventilation scans and chest $x$ ray examinations were performed two days before and five or six days after operation. The results were assessed visually by three independent observers who did not know the result of the F.U.T. The technique of assessment was similar to that previously used for liver scans and which has been found to be reproducible (Davies et al., 1972). The assessors agreed in all the cases diagnosed as pulmonary emboli.

\section{Results}

Of the 40 patients studied 11 (27.5\%) developed F.U.T. thrombi in their lower limbs after operation; 6 appeared on the first day, 4 on the third day, and 1 on the fifth day. All thrombi were confined to the calf.

Seven patients $(17.5 \%)$ had changes in the perfusion of their lungs without any changes in ventilation or the chest $x$-ray 
picture. The perfusion changes were in the lower zones in 4, in the apex in 1 , and in many segments in 2 . Only one patient had the physical signs of embolism. Another seven patients had changes in perfusion and ventilation and chest $x$-ray appearances consistent with a diagnosis of lung infection together with postoperative pyrexia and purulent sputum. All the perfusion changes in these seven were in lung bases.

Six patients had pyrexia, purulent sputum, and physical signs diagnostic of postoperative chest infection with no changes in the perfusion or ventilation scan. Thus 13 patients $(32.5 \%)$ were considered to have some degree of chest infection.

Of the 11 patients with F.U.T. thrombi $6(54.5 \%)$ had changes in lung perfusion alone and $5(45.5 \%)$ had no changes in lung perfusion. Only one of the 29 patients with normal legs had perfusion changes (see table). This high incidence of pulmonary emboli in patients with F.U.T. thrombi is highly significant $\left(x^{2}=14.5 ; \mathrm{P}<0.001\right)$.

Thus $85 \%$ of the patients with a diagnosis of pulmonary embolism had F.U.T. thrombi. Conversely over half of the patients with F.U.T. thrombi had emboli. Only one patient had changes in lung perfusion consistent with the diagnosis of cmbolism but no detectable thrombus in the legs.

Of the 13 patients with chest infection only 3 had an F.U.T. thrombus; the other 10 had normal legs. The incidence of chest infection in patients with F.U.T. thrombi (see table) was not significant $\left(x^{2}=0.2 ; 0.7>P>0.5\right)$. Indeed, there was a lower incidence of postoperative lung infections in the patients with thrombosis than in those with normal legs $(27 \%$ and $35 \%$ respectively).

Relation between "F.U.T. Thrombus" and Pulmonary Embolism and Pneumonia

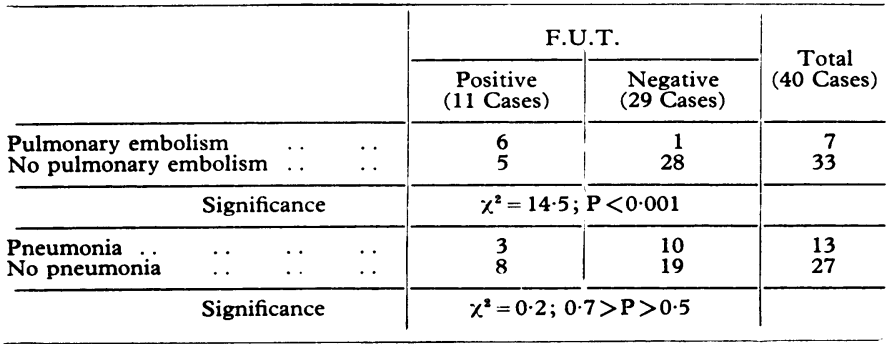

\section{Discussion}

Though this report is on relatively few patients the results are statistically significant and important. It is generally accepted that $25-30 \%$ of all patients undergoing general surgery in the U.K. develop small thrombi in their legs. This study shows that at least $18 \%$ of all patients undergoing general surgery have small pulmonary emboli, as evidenced by combined ventilation-perfusion scanning. The results rely on the high degree of accuracy of this technique in diagnosing pulmonary emboli (Williams et al., 1974).

Though necropsy studies have shown pulmonary embolism to be common this is the first study to show that emboli are common in healthy people who survive surgery. Of those who developed pulmonary embolism four-fifths had detectable thrombi in their legs; only one-fifth had normal legs. These studies suggest a definite connexion between the presence of F.U.T. thrombi in the legs and pulmonary embolism. It gives support to the suggestion that any prophylaxis which reduces the incidence of F.U.T. thrombi will reduce the incidence of pulmonary embolism.

This study has assumed that a change in lung perfusion in the absence of a change in ventilation and chest $x$-ray appearances indicates a loss of pulmonary artery blood flow due to an embolus, and evidence to this effect has been published (Williams et al., 1974). The value of our lung studies has been enhanced by obtaining them before and after operation. Six of the 40 patients studied had abnormalities of both perfusion and ventilation before operation, one had a perfusion defect only, and one had a ventilation defect. Without the preoperative studies these would have been misinterpreted.

Our patients had a high incidence of lung infection $(32.5 \%)$. Only half of these patients had perfusion and ventilation defects. Most patients with normal lung scans had transient episodes of pyrexia and basal moist sounds cured by physiotherapy. There was usually, but not always, a change in ventilation, perfusion, and chest $x$-ray appearances in those patients with persistent chest infection. Occasionally there were definite $x$-ray changes and no scanning abnormalities. We cannot say that the patients who developed pneumonia had not done so because of an underlying embolus but it is unlikely, as slightly fewer patients with F.U.T. thrombi developed chest infection than without F.U.T. thrombi.

Two questions arise from this study. If one accepts our findings and those of other studies on the incidence of F.U.T. thrombi then one must speculate that deep vein thrombosis and pulmonary embolism might be a normal or slight exaggeration of a normal physiological mechanism. Many believe that there is a balance in the blood between coagulation and thombolysis, and the frequent thromboemboli we now detect in surgical patients may result from a slight shift in this balance towards coagulation.

The second question is whether the mechanism that produces these thrombi and emboli is the same as the one that produces the large extending thrombus and massive embolism. An association between subclinical embolism and F.U.T. thrombi should not be taken to imply that preventing these thrombi will stop fatal pulmonary embolism but it makes it highly probable. It certainly suggests that any method of prophylaxis that reduces the incidence of F.U.T. thrombi will reduce the incidence of small emboli. We hope that our study of prophylaxis using the lung scanning techniques described will answer this question soon.

Requests for reprints should be sent to Professor N. L. Browse.

\section{References}

Browse, N. L., et al. (1971). British Medical fournal, 4, 325.

Ahmad, A. I. and Clapham, W. F., (1974). To be published

Davies, R. J., Cotton, P. B., Sweetland, C. A., Vernon, M., and Croft, D. N. (1972). Lancet, 1, 927.

Flanc, C., Kakkar, V. V., and Clarke, M. B. (1968). British fournal of Surgery, 55, 742 .

Negus, D.; Pinto, D. J., Le Quesne, L. P., Brown, N., and Chapman, M. (1968). British fournal of Surgery, 55,835 .

Sevitt, S., and Gallagher, N. (1961). British fournal of Surgery, 48, 475.

Wessler, S. (1962). American fournal of Medicine, 33, 648.

Williams, O., Lyali, J., Vernon, M., and Croft, D. N.'(1974). British Medical fournal, accompanying paper 